\title{
GROUND MOTION STUDIES FOR LARGE FUTURE LINEAR COLLIDERS
}

\author{
Shigeru Takeda, Noboru Yamamoto, and Katsunobu Oide \\ KEK, High Energy Accelerator Research Organization \\ Oho, Tsukuba, Ibaraki 305, Japan

\section{INTRODUCTION}

\section{LINAC AND GROUND MOTION}

The future large accelerator, such as a $\mathrm{TeV}$ linear collider, should have extremely small emittance to perform the required luminosity. Precise alignment of machine components is essential to prevent emittance dilution. The ground motion spoils alignment of accelerator elements and results in emittance growth. This paper discusses about ground motion and its effect on the main linac. The ground motion in the frequency range of seismic vibration is mostly coherent in the related accelerator. But the incoherent diffusive or Brownian like motion may become dominant at frequency region less than seismic vibration. Shiltsev reviewed many studies of ground motion and discussed about the diffusive motion [1]. Sery discussed about the ground motion focusing on the effects to the main linac of linear collider [2]. This paper intends to start from the power spectrum of the $A T L$ model [3], then show typical parameters of main linacs and consider measured data including excavated effects.

\section{POWER SPECTRUM OF $A T L$ MODEL}

Th ATL model can be formulated using an auto correlation function $\langle y(t+\tau) y(t)\rangle_{a v}$, as

$$
\begin{aligned}
\Delta_{y}(\tau)^{2} & =2\left\langle y(t)^{2}\right\rangle_{a v}-2\langle y(t+\tau) y(t)\rangle_{a v} \\
& =A \cdot L \cdot \tau .
\end{aligned}
$$

Here, $\langle X\rangle_{a v}$ means an ensemble average of $X$. Using the definition of a power spectral function,

$$
P(f)=\int_{-\infty}^{\infty} d f\langle y(t+\tau) y(t)\rangle_{a v} e^{-2 \pi i f \tau},
$$

Eq. 1 becomes,

$$
A \cdot L \cdot \tau=4 \int_{-\infty}^{\infty} d f P(f) \sin ^{2}(\pi f \tau) .
$$

If we assume that the power spectral function is proportional to the inverse of squared frequency,

$$
P(f)=\frac{K}{4 \pi^{2} f^{2}},
$$

we can carry out integration in the the right hand side of Eq. 3,

$$
4 \int_{-\infty}^{\infty} d f P(f) \sin ^{2}(\pi f \tau)=K \cdot \tau .
$$

Putting this result into Eq. 3, we find the power spectral function of $A T L$ model as,

$$
P(f)=\frac{A \cdot L}{4 \pi^{2} f^{2}} .
$$

Let us estimate emittance growth due to the drift in the transverse alignment of the accelerating structures caused by the $A T L$ model. Although the drift of the ground also affects the transverse alignment of the focusing elements, the orbit can be maintained as a straight line from the injection point to the extraction point with beam-based techniques. We assume that the orbit correction is so perfect that the orbit is an ideal straight line with an injection angle created by an achromat kicker placed at the entrance of the linac. Thus only the misalignment of accelerating structures is the source of the emittance blow-up in this case. Here we concentrate on the single bunch effect due to the dipole wake of the structures.

The transverse displacement of the beam at the end of linac caused by a misaligned accelerating structure $\delta y(s)$ is written as

$$
\begin{aligned}
& \Delta y_{1}(z)=\int d s \frac{N e^{2} w(z)}{E(s)} \\
& \quad \times \sqrt{\frac{\gamma(s)}{\gamma_{1}} \beta_{1} \beta(s)} \sin \left(\psi_{1}-\psi(s)\right)(\delta y(s)-y(s)),
\end{aligned}
$$

where $w(z)$ is the transverse wake function per unit length, $N$ the number of particles per bunch, $E(s), \gamma(s), \beta(s)$, $\psi(s)$ are the beam energy, Lorents factor, $\beta$-function, and betatron phase at location $s$, respectively. Suffices 0 and 1 denote the values of those functions at the entrance and the exit of the linac. We set the origin of the coordinate at the entrance of the linac as $s_{0}=0, y(0)=0$. The orbit $y(s)$ is always maintained to be linear in s, i.e.,

$$
y(s)=y_{1} s / L,
$$

where $L=s_{1}$ is the total length of the linac.

The expected value of square of the orbit is written as

$$
\begin{aligned}
& \left\langle\Delta y(z)^{2}\right\rangle=\left(\frac{N e^{2} w(z)}{E_{0}}\right)^{2} \int d s \int d s^{\prime} \\
& \times \frac{\beta_{1} \beta_{0} \gamma_{0}^{1 / 2}}{\gamma_{1}\left(\gamma(s) \gamma\left(s^{\prime}\right)\right)^{1 / 4}} \sin \left(\psi_{1}-\psi(s)\right) \sin \left(\psi_{1}-\psi\left(s^{\prime}\right)\right) \\
& \times\left\langle(\delta y(s)-y(s))\left(\delta y\left(s^{\prime}\right)-y\left(s^{\prime}\right)\right)\right\rangle,
\end{aligned}
$$

where we have assumed a scaling of focusing as $\beta(s)=$ $\beta_{0} \sqrt{\gamma(s) / \gamma_{0}}$. The $A T L$ model gives the correlation of displacements between at $s$ and $s^{\prime}$ or $s$ and $s_{1}$ :

$$
\begin{aligned}
\left\langle\delta y(s) \delta y\left(s^{\prime}\right)\right\rangle & =A t \times \min \left(s, s^{\prime}\right) \\
\left\langle\delta y(s) y_{1}\right\rangle & =A t s .
\end{aligned}
$$


The betatron phase is expressed as

$$
\psi(s)=\int_{0}^{s} \frac{d s}{\beta(s)}=\frac{2 \sqrt{\gamma_{0}}}{g \beta_{0}}\left(\sqrt{\gamma(s)}-\sqrt{\gamma_{0}}\right),
$$

where $g$ is the acceleration gradient $g \equiv d \gamma / d s$. Putting Eqs. 8, 10, and 11 into Eq. 9, we obtain the expected value of the relative blow-up of the beam as

$$
\begin{aligned}
\epsilon & \equiv \frac{\left\langle\Delta y(z)^{2}\right\rangle}{\left(\beta_{1} \varepsilon_{N y} / \gamma_{1}\right)} \\
& \approx A t \frac{N^{2} e^{4} w(z)^{2}}{4 g^{2} E_{0}^{2} L \varepsilon_{N y}} \beta_{0}^{3} \gamma_{0}^{1 / 4} \gamma_{1}^{11 / 4}
\end{aligned}
$$

where $\varepsilon_{N y}$ is the invariant emittance. We have approximated $\left(\gamma(s) \gamma\left(s^{\prime}\right)\right)^{1 / 4} \approx\left(\gamma_{0} \gamma_{1}\right)^{1 / 4}$, and have assumed

\begin{tabular}{|c|c|c|c|c|}
\hline & & $\overline{\mathrm{X} \text {-banc }}$ & $\overline{\mathrm{C}-\mathrm{ban}}$ & \\
\hline $\begin{array}{l}\text { Beam energy } \\
\text { entrance/exit }\end{array}$ & $E_{0} / E_{1}$ & \multicolumn{2}{|c|}{$10 / 500$} & $\mathrm{GeV}$ \\
\hline Particles/bunch & $N$ & 0.8 & 1.0 & $10^{10}$ \\
\hline Inv. emittance & $\varepsilon_{N y}$ & \multicolumn{2}{|c|}{30} & $\mathrm{~nm}$ \\
\hline Bunch length & $\sigma_{z}$ & 80 & 200 & $\mu \mathrm{m}$ \\
\hline$\beta$ at entrance & $\beta_{0}$ & \multicolumn{2}{|c|}{4} & $\mathrm{~m}$ \\
\hline Rf frequency & $f$ & 11.4 & 5.7 & $\mathrm{GHz}$ \\
\hline Acc. gradient & $d E / d s$ & 45 & 32 & $\mathrm{MeV} / \mathrm{m}$ \\
\hline $\begin{array}{l}\text { Iris radius/ } \\
\text { Wavelength }\end{array}$ & $a / \lambda$ & 0.16 & 0.14 & \\
\hline$A T L$ coeff. & $A$ & \multicolumn{2}{|c|}{1} & $\mathrm{~nm}^{2} / \mathrm{m} / \mathrm{s}$ \\
\hline $\begin{array}{l}\text { Stable time } \\
\text { for } \epsilon=0.1\end{array}$ & $t$ & 3 & 24 & hours \\
\hline
\end{tabular}
$g \beta \gg 1$, etc.

TABLE 1: Parameters of typical linear collider linacs.

Equation 12 gives the duration time $t$ for the emittance blow-up remains within an amount $\epsilon$. The actual magnitude of $t$ thus depends on the parameters of the linac. As an example we calculate for two cases of X-band and C-band. We assume the focusing parameters are same in two cases. We applied an approximation by K. Yokoya for the wake function $w(z)$ to calculate the blow-up factor $\epsilon$ in Eq. 12:

$$
w(z) \approx w^{\prime} \sigma_{z} \approx 1.55 \frac{c Z_{0}}{\pi a^{4}}\left(\frac{a / \lambda}{0.14}\right)^{0.179} \sigma_{z}
$$

where $a, \lambda, Z_{0}$ are the iris radius, the $\mathrm{rf}$ wavelength, and the vacuum impedance, respectively. The parameters in Table 1 may be different from existing designs in detail, but the essential characteristics are not much far from "real" designs.

\section{PROPERTIES OF GROUND MOTION}

In the frequency range $f<0.1 \mathrm{~Hz}$, the measured power spectrum of ground motion can be characterized by $k / f^{2}$. This coefficient $k$ has site dependence and changes from 1 to $10^{2} \mathrm{~nm}^{2} / H z$. The sources of the ground motion are the atmospheric activity, change of underground water, ocean tide, earth-tides, temperature variation of the surface ground etc $[5,6,2]$. At high frequency $f>0.1 \mathrm{~Hz}$, the power spectrum decreases very rapidly with frequency being proportional to $1 / f^{4}$. The motion consists of seismic activity, that is elastic motion. Correlation measurements show that the motion has good correlation in quiet sites [6, 2].

The motion at $f<0.1 \mathrm{~Hz}$, however, is very different. Dominant part of the ground motions is inelastic motion, though the elastic motion presents in this frequency range. The earth-tides, one of the elastic motion in this low frequency range, becomes cause of inelastic motion through its dissipation. One of the models describing this inelastic ground motion is the $A T L$ model mentioned above [3]. The $A T L$ model means that slow relative ground motion is random and the number of discrete breaks appearing between two points is proportional to the distance and the passed time. These discrete breaks consist of random popping up/down of fragmented rocks. The duration times and the amplitudes of popping motion of the rocks are not complete random phenomena, then this two-dimensional covariance function and the white noise correlation are not equivalent. The $A T L$ model is one of the mathematical approximation for this covariance function provided the process of random motion including slow variable component. The parameter $A$ should be influenced dominantly by the earth properties, providing the source of inelastic motions is dissipation of the elastic motion. In other words, the value of $A$ should depend on the degree of fragmentation of the rock. Observed parameter $A$ is smaller in the tunnel of solid rock than in the broken rock [1]. The excavation

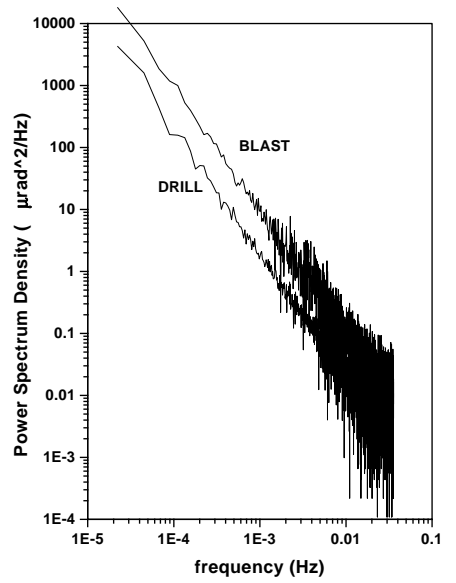

FIGURE 1: Power spectrum densities in the tunnel excavated by Drill or Blast.

method of the tunnel also affects significantly the value of $A$, as shown in Fig. 1 [5]. Although the tunnel excavated by use of dynamites gives $A \cong 5 \mathrm{~nm}^{2} / \mathrm{m} / \mathrm{s}$, the tunnel bored by a machine gives $A \cong 1 \mathrm{~nm}^{2} \mathrm{~m} / \mathrm{s}$. This difference shows probably because of artificial fragmentation of the rock occurred during construction. Our experimental results and recent report on the excavation clarify that application of TBMs is better excavating method than NATM 
(Drill + Blast) for accelerator tunnel to prevent emittance dilution $[5,7]$.

NATM (New Austrian Tunnelling Method) is a excavating method with distributed dynamites and drills. Many rockbolts are essential to prevent relaxation of the rock on NATM. TBM (Tunnel Boring Machine) is a name of tunneling tool which is a very big drilling machine. Excavation using TBMs in the massive hard rock eliminates overbreak on the surface of the tunnel and no tunnel support may be possible. On the other hands, NATM gives relaxation in the surface layer of the rock caused by blast, then utilization of many rockbolts becomes essential to support the surface [7]. Fragmentation of the rock through this relaxation gives the increase in $A$.

TABLE 2: Typical values of $A$ in the $A T L$ model

\begin{tabular}{lr|ll}
\multicolumn{3}{c}{$A: \mathrm{nm}^{2} / \mathrm{m} / \mathrm{s}$} \\
\hline \hline \multicolumn{2}{l|}{ Laboratory Site } & \multicolumn{2}{|c}{ Other Site } \\
\hline UNK & 100 & Pinon Flat & 0.1 \\
SLAC & 100 & Esashi & 0.3 \\
CERN & 10 & Sazare & 0.05 \\
KEK & 40 & & \\
\hline \hline
\end{tabular}

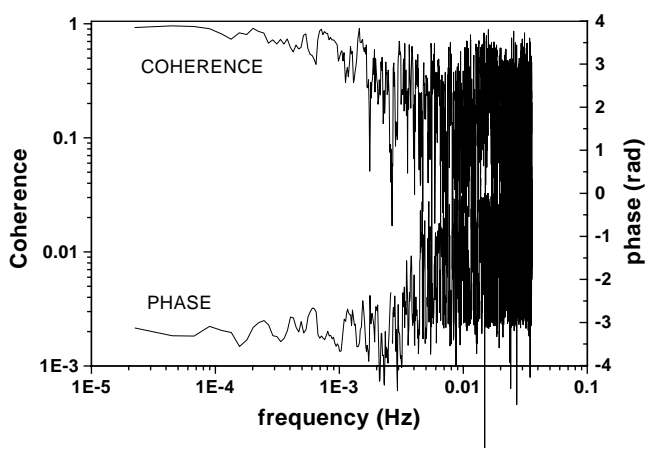

FIGURE 2: Coherence and phase difference obtained in the tunnel excavated by NATM. Two levels were set up 46 meters apart.

The tolerance for errors of the linear collider strongly depends on the design of the machine. We summarize the typical data of $A$ in Table 2, in order to clarify the severe tolerance of the machine. The natural level of seismic vibrations has been usually considered as seriously affecting machine operation. They are assuming that slow ground motion much less than characteristic frequency of an accelerator gives complete space and time coherence of the machine displacements. Our experimental results, however, suggests being frequency dependent structure for the space coherence in the low frequency range [5]. We have observed the slow ground motion in the tunnels which were excavated using different methods. Figures 2 and 3 show the coherence and phase difference between the two points separated by a distance $46 \mathrm{~m}$. The followings are found out from the figures:
1. The parameter $A$ is found about 5 times difference.

2. The coherence decreases in the frequency range of $5 \times$ $10^{-4} \mathrm{~Hz}$ or more in Fig. 2, on the other hand, Fig. 3 shows good coherence for almost all frequency range.

3. The phase relation has a big difference between them. In Fig. 2, the phase changes in the whole frequency range and it becomes at random in the frequency range of $3 \times 10^{-3} \mathrm{~Hz}$ or more, though it is almost stable in Fig. 3. Out of phase in the low frequency range of Fig. 2.

4. The tunnel is divided into the block and our obtaining result is information on the movement of the block.

5. The size of the block depends on the the method of excavating the tunnel and/or geological property.

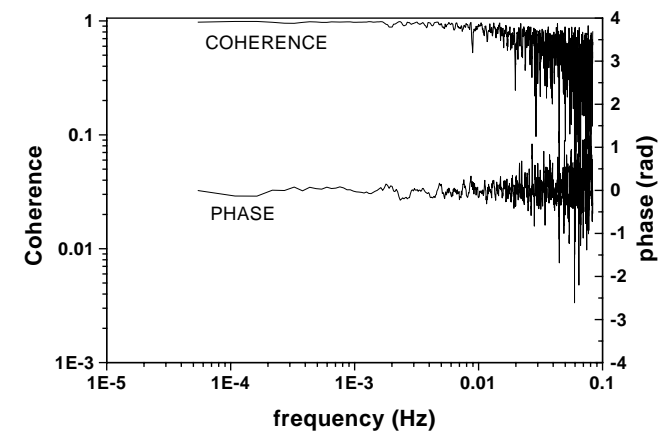

FIGURE 3: Coherence and phase difference obtained in the tunnel excavated by TBM. Two levels were set up 46 meters apart.

\section{REFERENCES}

[1] V. Shiltsev 'Space-Time Ground Diffusion: Space- Time Ground Diffusion: The ATL Model for Accelerators', Proc. IWAA95, KEK, Nov. 14-17 (1995) pp. 352-381. Many references are there.

[2] A. Sery 'Ground Motion Studies with Respect to Linac Performance', Proc. of LINAC96, CERN96-07 pp. 621-625.

[3] B. Baklakov, P. Lebedev, V. Parkhomchuk, A. Sery, A. Sleptov and V. Shiltsev, INP 91-15; Tech. Ph. 38, 894, (1993).

[4] K. Yokoya, private communications.

[5] Shigeru Takeda et al., Proc. of EPAC96, 1996. ; S. Takeda, K.Kudo, Y. Funahashi, A. Akiyama and Y. Kanazawa 'Slow Ground Motion and Alignment System', Proc. EPAC94, London, June 3 (1994) pp. 2564-2566; S. Takeda and M. Yoshioka 'Ground Motion at KEK', KEK Preprint 95-209 Feb. 1996.

[6] S. Takeda, A. Akiyama, K.Kudo, H. Nakanishi and N. Yamamoto 'Slow Drift and Frequency Spectra on Ground Motion', Proc. 3rd Int. Workshop on Accelerator Alignment, Sept. (1993) pp. 225-232.

[7] P. J. Tarkoy 'Comparing TBMs with Drill+Blast Excavation', Tunnels and Tunnelling Oct. (1995) pp41-44. 